\title{
El surgimiento del Teatro Popular José González Castillo a partir del análisis de su documentación interna
}

\section{The Emergence of the Teatro Popular José González Castillo according to the Analysis of its Internal Documentation}

Artículo recibido el 9 de abril de 20I8; devuelto para revisión al 6 de septiembre de 20I8; aceptado el I9 de noviembre de 20I8, http://dx.doi.org/IO.220I/iie.18703062e.2019.II4.2668

María Fukelman Instituto de Artes del Espectáculo, Facultad de Filosofía y Letras, Universidad de Buenos Aires/Consejo Nacional de Investigaciones Científicas y Técnicas/Universidad Católica Argentina, mariafukelman@gmail.com

Líneas de investigación Teatro argentino, historia del teatro, teatro independiente.

Lines of research Argentine Theater, Theater History, Independent Theater.

Publicaciones más relevantes Compilación y participación autoral en el libro Teatro independiente. Historia y actualidad (Buenos Aires: Ediciones del CCC, 20I7); "Los inicios del teatro independiente en Buenos Aires y su vínculo con la macropolítica", Anagnórisis. Revista de Investigación Teatral, núm. I6 (2017): I05-I29.

Resumen El teatro independiente surgió en Buenos Aires a comienzos de la década de 1930. Entre sus antecedentes, se encuentra la polémica literaria de Boedo y Florida, grupos opuestos en la que los intelectuales buscaban "el arte para la revolución" y "la revolución para el arte", respectivamente. El dramaturgo José González Castillo fue creador de la Peña Pacha Camac. A través del análisis de documentación original, abordaré el surgimiento de la Peña, su conformación institucional y la constitución de su grupo de teatro.

Palabras clave Teatro independiente; Buenos Aires; Boedo; documentación; González Castillo; Peña.

\begin{abstract}
Independent theater emerged in Buenos Aires at the beginning of the 1930's. Among its antecedents, the literary polemic between Boedo and Florida can be found; opposing groups in which intellectuals, sought an "art for the revolution" or "the revolution for art". The playwright, José González Castillo, created the Peña Pacha Camac. Through the analysis of original documentation, I will review the materialization of the Peña, its institutional conformation, and the constitution of its theater group.
\end{abstract}

Keywords Independent theater; Buenos Aires; Boedo; documentation; José González Castillo; Peña. 


\section{MARÍA FUKELMAN \\ instituto DE ARTES Del espectáculo,}

FACULTAD DE FILOSOFÍA Y LETRAS, UNIVERSIDAD DE BUENOS AIRES/ CONSEJO NACIONAL DE INVESTIGACIONES CIENTÍFICAS Y TÉCNICAS/

UNIVERSIDAD CATÓLICA ARGENTINA

ARGENTINA

\section{El surgimiento del Teatro Popular José González Castillo a partir del análisis de su documentación interna}

$\mathrm{L}$ a crítica especializada coincide en considerar que el teatro independiente se instaló en Buenos Aires a partir de la iniciativa del escritor y periodista Leónidas Barletta, y la fundación de su Teatro del Pueblo, que data del 30 de noviembre de 1930. En mi reciente tesis doctoral, ${ }^{1}$ lo he definido como un modo de producir y de concebir el teatro que pretendió renovar la escena nacional de tres maneras: se diferenció del teatro que ponía los objetivos económicos por delante de los artísticos; se propuso realizar un teatro de alta calidad estética; careció de fines lucrativos. En este sentido, se constituyó como una práctica colectiva y contestataria — dado que se opuso al statu quo del teatro de aquellos años e impulsó una organización anticapitalista-, pero también heterogénea, ya que se mostró como un enraizado complejo y rico en su diversidad.

Los motivos de la iniciación del teatro independiente fueron varios, no hubo una única razón, sino que resultó de una combinación de factores. Uno de los

I. María Fukelman, "El concepto de 'teatro independiente' en Buenos Aires, del Teatro del Pueblo al presente teatral: estudio del periodo I930-1944", tesis de doctorado (Buenos Aires: Universidad de Buenos Aires-Facultad de Filosofía y Letras, 20I7). 
más importantes fue el objetivo de distanciarse de la escena del teatro profesional (en la que abundaban revistas, sainetes y vaudevilles), cuyas características no agradaban a Barletta ni a muchos de sus colegas de la época. ${ }^{2}$ Otro motor para transformar el teatro de Buenos Aires fue que los artistas y escritores, que luego darían forma al movimiento de teatros independientes, tenían cierto conocimiento (a través de las revistas culturales) sobre las novedades de la vanguardia artística que estaban sucediendo en Europa.

Asimismo, en la década que precedió a la conformación de este movimiento, muchos de los intelectuales de Buenos Aires se habían dividido en dos grupos: Boedo y Florida. El primero, cuyo nombre surgió de la calle donde funcionaba la Editorial Claridad (Boedo 837), abogó por un arte social, de temática directa, que fuera accesible a los obreros y que los invitara a accionar. El segundo, que también debió su nombre a la calle, elegante y céntrica, donde hacía sus ediciones, pretendía renovar la literatura con fines puramente estéticos. Las trincheras literarias desde las que cada grupo expresaba su mirada eran el periódico Martín Fierro (1924-1927), dirigido por Evar Méndez, para los de Florida; y las revistas Los Pensadores ${ }^{3}$ (1924-1926) y Claridad. Tribuna del Pensamiento Izquierdista (1926-194I), dirigidas por Antonio Zamora, para el grupo Boedo. Aunque - a grandes rasgos - los de Florida buscaran "la revolución para el arte" y los de Boedo se inclinaran por "el arte para la revolución", en el contexto geopolítico que los circundaba (pocos años habían pasado desde la Revolución de Octubre, de 1917, que abrió el camino para la creación de la Unión de Repúblicas Socialistas Soviéticas, URSS, en 1922), ambos bandos celebraron la emancipación de la clase obrera.

Tradicionalmente, se ha dicho que el teatro independiente es heredero del grupo de Boedo, dado que Leónidas Barletta integraba este conjunto (había sido secretario de redacción de Claridad). Además, allí se enunció por primera

2. Véase Hipólito Carambat, "El Teatro en Buenos Aires", Revista Martín Fierro 1924-1927, edición facsimilar, est. prel. Horacio Salas (Buenos Aires: Fondo Nacional de las Artes, 1995): 17; Armando Eneas, "El arte y el pueblo", Los Pensadores, núm. II7 (enero 1926): s.p.; E. Morfino, "El teatro y sus intérpretes", Los Pensadores, núm. Io9 (I4 de abril de 1925): s.p; Leónidas Barletta, "Sobre teatro nacional", Claridad. Revista de Arte, Critica y Letras. Tribuna del Pensamiento Izquierdista, núm. I34 (I5 de mayo de 1927): s.p; y Álvaro Yunque, "Nuestro teatro", Claridad. Revista de Arte, Critica y Letras. Tribuna del Pensamiento Izquierdista, núm. 136 (Io de junio de I927); entre otros.

3. Los Pensadores existía desde 1922 con otro formato: cada número estaba dedicado a un pensador y se publicaba una de sus obras. 
vez (según mi registro) la intención de conformar un teatro independiente. ${ }^{4}$ Sin embargo, hay elementos del grupo de Florida que también se pueden considerar contribuidores al desarrollo de esta práctica teatral. A su vez, ambos conjuntos coincidían en algunos puntos, como en la necesidad de renovar el teatro. ${ }^{5}$ Estas expresiones publicadas en los años veinte dan cuenta de que las ideas estaban siendo pensadas y elaboradas mucho tiempo antes de que pudieran llevarse a cabo. Los cambios no se produjeron de un día para el otro ni fueron producto del trabajo de una sola persona, sino que comenzaron a vislumbrarse en forma paulatina. Los primeros grupos que se conformaron (Teatro Libre, 1927; Teatro Experimental de Arte, 1928; La Mosca Blanca, 1929, y El Tábano, 1930) pretendieron, aunque sin demasiado éxito, llevar a la práctica estas teorías.

También de Boedo, pero del barrio más que del grupo literario, fue José González Castillo. Allí creó la Peña Pacha Camac, cuyo elenco de teatro devino, luego de su muerte, en el Teatro Popular José González Castillo, teatro independiente al que me referiré en el presente trabajo. En primer lugar, abordaré su surgimiento y conformación institucional, por medio del análisis de documentación original. Mucha de la información que presentaré se extrajo de las actas de la Peña de Artistas Pacha Camac (tal era su nombre completo), que pude consultar en el archivo de la Junta de Estudios Históricos del barrio de Boedo. Esta documentación consta de:

- El acta fundacional de la peña, del 30 de julio de 1932;

- Una parte del acta de la asamblea general de agosto de 1932;

- Las primeras once actas, redactadas entre el 28 de septiembre de 1932 y el 29 de mayo de 1933 ;

- 198 hojas consecutivas de las actas llevadas por los integrantes de la peña entre el 30 de octubre de 1937 y el 6 de noviembre de 1943 .

Además, cuento con bibliografía pertinente. ${ }^{6}$

4. Véase Anónimo, "Algo más sobre el teatro", Claridad. Revista de Arte, Crítica y Letras. Tribuna del Pensamiento Izquierdista, núm. I3I (marzo 1927): s.p.

5. Véase María Fukelman, "Los antecedentes del teatro independiente en Buenos Aires: la importancia de Boedo y Florida", Culturales, vol. 5, núm. I (enero-junio 2017): I5I-I87.

6. Véase Osvaldo Carlos Sidoli, Los literatos de Boedo y la Peña "Pacha Camac" (Buenos Aires: Rotary Club de Boedo, 1995); Catalina Artesi, "Boedo y el teatro independiente", en Primer Congreso de Historia del Barrio de Boedo (Buenos Aires: Junta de Estudios Históricos del Barrio de Boedo, 1997), 15-20; Aníbal Lomba, Peña Pacha Camac 1932-1957. Una peña nacida en Boedo 
Asimismo, es interesante destacar que este barrio de la Ciudad Autónoma de Buenos Aires continúa siendo un núcleo cultural que conserva la memoria sobre su célebre pasado. La mencionada Junta de Estudios Históricos del Barrio de Boedo - institución a la que le agradezco su apoyo- ha organizado congresos sobre el barrio, ha colocado placas y bustos de figuras representativas en distintas arterias del barrio y se ha encargado de difundir material informativo. A su vez, el edificio sobre la avenida Boedo — donde se encontraba el Café Biarritz y, dentro de él, la Peña Pacha Camac — es hoy un museo del Banco Ciudad y allí se reconstruye no sólo la historia del banco, sino también la del tradicional café, por lo que en sus paredes se puede ver, entre otras reliquias, el acta de fundación de la Peña Pacha Camac.

\section{Los inicios de la Peña de Artistas Pacha Camac}

La Peña de Artistas Pacha Camac, instalada en la terraza del Café Biarritz, en Boedo 868, fue creada en julio de 1932 por José González Castillo. Para hacerlo, el dramaturgo, director, poeta y docente — quien, para ese entonces, ocupaba un lugar reconocido en la escena de teatro profesional desde hacía más de veinte años - contó con la ayuda de su amigo Julio Cruciani, y con el apoyo económico y el trabajo de los vecinos y comerciantes de Boedo. Algunos años antes (1928), González Castillo había fundado, a pocas cuadras de allí, la Universidad Popular de Boedo (hoy es una escuela pública, la núm. 22, "Martina Silva de Gurruchaga"), destinada a los trabajadores de la zona.

El nombre de la peña remite al de un dios de la mitología inca considerado el creador del mundo. Mónica Villa ${ }^{7}$ supone que González Castillo eligió este mote andino por haberse codeado con la cultura incaica durante su educación en el Seminario de Orán, en la provincia de Salta, donde fue criado por un tío sacerdote, luego de haberse quedado huérfano.

En la Peña Pacha Camac se formó el primer grupo de teatro de Boedo, que comenzó siendo filodramático. Los cuadros filodramáticos eran "grupos formados por voluntariosos vecinos, de inestable continuidad, que con esfuerzo $\mathrm{y}$, por lo general, escasa idoneidad artística, desde principios del siglo xx, se

para toda la Ciudad (Buenos Aires: Junta de Estudios Históricos del Barrio de Boedo, 2010); y Mónica Villa, José González Castillo. Militante de lo popular (Buenos Aires: Corregidor, 2015).

7. Villa, José González Castillo. Militante de lo popular. 
reunían en clubes de barrio, salas de sindicatos, sociedades de fomento o centros políticos para llevar adelante un proyecto teatral que casi nunca superaba una única función". ${ }^{8} \mathrm{La}$ diferencia fundamental entre éstos — muchos de los cuales existían antes que el Teatro del Pueblo- y los grupos de teatro independiente fue que los primeros imitaban el contenido y las formas del teatro profesional sin pretender dar cuenta de procesos creativos propios. Además, los elencos filodramáticos no solían reflexionar sobre el teatro, ni plantear una posición respecto al "negocio teatral”, ni asumir una ideología sobre la función del arte, entre otras cuestiones.

Si bien, José Marial ${ }^{9}$ sostuvo que fue en 1934 el momento en el que el elenco de teatro adquirió autonomía dentro de la peña, esto no sucedió sino hasta I937, luego de la muerte de su director. Allí el grupo aprehendió su denominación definitiva, Teatro Popular José González Castillo, y se asentó como un teatro independiente.

La Peña de Artistas Pacha Camac contaba con un escenario, camerinos y una sala con capacidad para 300 espectadores. Para la construcción del modesto local se utilizó madera, cinc, cartón y papel. Funcionó como un múltiple espacio cultural, desarrollando en el barrio de Boedo - lejos del tradicional centro porteño - una actividad sin precedentes: brindó representaciones teatrales, conciertos, exposiciones, conferencias, ${ }^{\mathrm{IO}}$ seminarios y cursos de teatro, declamación, música, pintura, escultura y dramaturgia, entre otros; también confeccionó una biblioteca y una hemeroteca. Para Catalina Artesi, "la educación popular a través del arte era el principal objetivo", ${ }^{\text {II }}$ de modo que las diversas clases no sólo eran gratuitas, sino que a los alumnos también se les brindaban los materiales de estudio y trabajo. Además, luego de varios intentos frustrados, la agrupación editó, desde 1943, una publicación propia titulada Pacha Camac. Boletín de la Peña de Artistas de Boedo "Pacha Camac", que tuvo, al menos, siete números, distribuidos en ocho años. Como afirma Mónica Villa, la Pacha Camac llegó a ser "uno de los espacios culturales más importantes que tuvo la

8. Roberto Perinelli, "El teatro independiente argentino: Una síntesis de su historia”, en comp. Cristina Quiroga, Actas del V Congreso Argentino Internacional de Teatro Comparado "Teatro latinoamericano y teatro del mundo" (Entre Ríos: Leviatán, 20I4), 37.

9. José Marial, El teatro independiente (Buenos Aires: Ediciones Alpe, 1955).

Io. Disertaron, entre otros, Roberto Arlt, Carlos Castagnino, Alicia Moreau de Justo, Alfredo L. Palacios, Marcelo T. de Alvear y Raúl González Tuñón.

II. Catalina Artesi, "Boedo y el teatro independiente", en Primer Congreso de Historia del Barrio de Boedo, I6. 
ciudad y abrió las puertas a una franja de la población para quienes el acceso al mundo del arte y del conocimiento habría sido imposible de no mediar el espíritu docente e integrador de la peña". ${ }^{2}$

\section{Los estatutos de la Peña Pacha Camac}

Las peñas eran sitios de reunión y entretenimiento muy frecuentes en Buenos Aires durante las décadas de los años veinte y treinta. El dramaturgo Vicente Martínez Cuitiño observó que se desarrollaban en algunos cafés, donde "al margen de las actividades de su dueño y de la corriente general del público consumidor, artistas e intelectuales fundaban su más cómodo club: un club sin reglamentos ni disciplinas ni responsabilidad, como no sea la tangencial e inesperada que la crónica pueda depararle". ${ }^{\text {I3 }}$

A pesar de esta forma de ser - libre - de las peñas, la Pacha Camac tuvo un acta fundacional, escrita por "artistas y amigos de las artes". Según Osvaldo Sidoli, este documento "es casi el certificado de la pobreza inicial de ese grupo de boedenses, empujados por sueños románticos: como no tenían mejor papel, pidieron al dueño del café una hoja en blanco que incluye el membrete

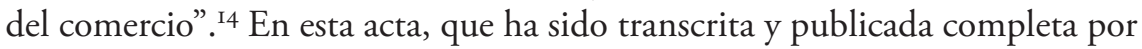
Aníbal Lomba, se dejaron asentados los principales objetivos de la asociación:

I) Fundar un sitio de reunión y camaradería en el barrio, para los pintores, escultores, músicos, escritores y aficionados a las actividades intelectuales y artísticas;

2) Propender a la difusión y al culto de las bellas artes en todo lo que se llama el barrio de Boedo, estimulando la obra, la cultura y el progreso moral de la juventud;

3) Realizar exposiciones y certámenes artísticos, conferencias y espectáculos públicos de finalidad cultural, y propiciar todo movimiento que tienda al progreso de las artes en el barrio y a la independencia moral y económica del artista. ${ }^{15}$

Este extracto del acta fundacional muestra la pretensión de conformar un centro cultural íntegro, expectativa que también se estaba avizorando en varios de

I2. Villa, José González Castillo. Militante de lo popular, I6I.

13. Citado en Villa, José González Castillo. Militante de lo popular, I6o.

14. Sidoli, Los literatos de Boedo y la Peña "Pacha Camac", 6-7.

15. Citado en Aníbal Lomba, Peña Pacha Camac 1932-1957. Una peña nacida en Boedo para toda la Ciudad, I9. 
los espacios que luego se convertirían en reconocidos teatros independientes, como el Teatro del Pueblo o el idramst (Idishe Dramatishe Stude -Estudio Dramático Judío-, germen del IFT, Idisher Folks Teater - Teatro Popular Judío-). Además, la Pacha Camac se asemejó a estos conjuntos en su certeza de que el arte podía transformar a la sociedad positivamente, es decir, hacerla progresar. Pero el elemento novedoso que destaca ahí, en relación a los teatros antes nombrados, es la idea de constituirse como un sitio de reunión y camaradería, evidenciando características menos rígidas. Con estas declaraciones, los artistas de la peña se acercaban a la visión del teatro como "entretenimiento" que se le atribuía al teatro profesional o "comercial", circuito con el que, como se verá más adelante, sostenían un vínculo estrecho. Por otro lado, así como el IFT se presentaría como un teatro centrado en la comunidad judía, la Peña Pacha Camac se concibió como un elemento propio del barrio de Boedo: era inseparable de su entorno. Asimismo, la frase de este documento que cataloga al artista como independiente moral y económicamente, vuelve a acercar a la peña al movimiento de teatros independientes que se estaba gestando a su alrededor.

En el acta fundacional, a continuación de lo ya citado, se designó una comisión organizadora (compuesta por Ernesto Lío y Domingo Perriello) para que redactara el estatuto de la agrupación, propusiera su nombre y las comisiones que la integrarían. Esta propuesta debía estar lista en los siguientes is días. Los firmantes del acta fundacional fueron José González Castillo, C. Floriani, Agustín Riganelli, Vicente Roselli, R. Ranone, Pablo Antonio Biscardi, Ernesto Lío, Rosario Caputto, Rafael Bertugno, Domingo Perriello, E. Aramburu, Juan Tuarbisetti, M. Petrone, Severo Renis, Juan Dastugne, Julio Giardullo, Gerónimo Caputto, Enrique Montero, Carlos Rohde y Vicente Riccio.

En el acta de la asamblea general, efectuada en agosto de 1932, se aprobaron los estatutos de la institución, y se hicieron algunas modificaciones sobre la proposición de Lío y Perrielo. Si bien no dispongo de la copia de los estatutos, es a través de estas modificaciones que es posible conocer algunos de sus artículos:

Art. I $^{\circ}$ La peńa Boedo se llamará Peńa de Artistas Pacha Camac.

Art. $2^{\circ}$ - Se reemplaza por otro que dice: "que en el momento oportuno se gestionará la personería jurídica”.

Art. $3^{\circ}$ - El capital social estará compuesto por: la cuota de los socios, ingresos extraordinarios, muebles e inmuebles. 
Art. $4^{\circ}$ - Los socios serán activos, cooperadores y honorarios. Los cooperadores serán los que contribuyan al sostenimiento de la peńa con una contribución especial; los honorarios, las personas que a juicio de la C.D. merezcan por sus méritos formar parte de la institución.

Art. $8^{\circ}$ - Los socios activos pagarán cincuenta centavos mensuales.

Art. $9^{\circ}$ - El socio que no abone seis meses será separado de hecho de la institución.

Art. $14^{\circ}-$ El socio que reingrese pagará sus cuotas atrasadas. ${ }^{\mathrm{I}}$

Hacia el final de la página, que continúa en otra que no tengo, se enuncia que los artículos $12^{\circ}, 13^{\circ}, 17^{\circ}$ y $18^{\circ}$ se suprimirán, y que la Comisión Directiva será ejercida por siete miembros. Su mandato será por dos años pero cada año se renovará por mitades, en cantidades de cuatro y tres alternativamente. La Comisión Directiva tendrá quórum con cuatro de sus miembros.

Más adelante, hay otros apartados en las actas en los que se vuelve sobre los estatutos. Por ejemplo, esto sucede en la Asamblea Extraordinaria del I6 de enero de 1933, cuando "el Sr. González Castillo apoyado en el hecho, de que la institución no marcha de acuerdo a las esperanzas en ella depositadas, por divergencias que entorpecen la labor de la Comisión Directiva, presenta su renuncia y pone a consideración de la asamblea la necesidad de reorganizar la institución". ${ }^{17}$ Este hecho genera un debate sobre si se le debe aceptar la renuncia al presidente y si hay que cambiar — O no- a toda la Comisión Directiva. Algunos miembros sostienen que, según las disposiciones del estatuto, la asamblea no reúne la mayoría necesaria para su validez pero, ante esto, la Secretaría informa que no existen los estatutos definitivos. Esto es llamativo, ya que, como se ha visto inicialmente, los estatutos habían sido aprobados un ańo antes. Acto seguido, se decidió, por mayoría, designar una comisión reorganizadora de la peña — con José González Castillo manteniéndose en la presidencia—, que tendría a su cargo la elaboración de los estatutos.

Ocho días más tarde, el 24 de enero de 1933, en una asamblea ordinaria se lee - pero no se transcribe en actas - un proyecto de estatutos. Sin embargo, se evidencia que este proyecto tampoco fue irreversible porque, en la Asamblea Provisoria del 29 de mayo de 1933, el presidente informa sobre la necesidad de

16. Archivo Junta de Estudios Históricos del Barrio de Boedo, Actas, Peña Pacha Camac, agosto de 1932 (extracto).

I7. Archivo Junta de Estudios Históricos del Barrio de Boedo, Actas, Peña Pacha Camac, i6 de enero de 1933. 
confeccionar los estatutos y de elegir una Comisión Directiva. Ahí, de nuevo, queda asentada la exposición de un proyecto de estatutos. Éste es discutido en general y en particular, y se le hace una modificación numérica: la Comisión Directiva estará integrada por I4 socios activos y cuatro adherentes. En esa asamblea, la comisión reorganizadora fija fecha para realizar una Asamblea Ordinaria para el día II de junio de I933. En dicha cita se tratarían los estatutos. Lamentablemente, esta acta de finales de mayo es la última del año I933 con la que cuento. Luego hay un salto temporal en la documentación hasta 1937.

No obstante, el tema de los estatutos continuará vigente varios años más. El Is de agosto de 1938, se informa que el grupo de arte escénico ha nombrado sus propias autoridades y se resuelve que el Teatro Popular José González Castillo (ya había adquirido su nombre definitivo) lleve un libro de actas con sus sesiones. En el renglón sucesivo, se decide confeccionar un proyecto de estatutos y se nombra una comisión para que se ocupe del tema e informe - en la siguiente reunión - sobre su trabajo. Según Lomba, tras la muerte de González Castillo, se hizo necesaria una estructura más formal que implicó la redacción de "un nuevo estatuto que reemplace la organización transitoria dada pocos años antes". ${ }^{8}$

El día 29 de agosto de 1938 se le dio entrada a los proyectos de estatutos presentados por la comisión encargada de hacerlos. El is de octubre, en la Asamblea Extraordinaria, los estatutos figuran en el número 2 de la orden del día y se enuncia: "A continuación se leen los estatutos confeccionados por la Junta Reorganizadora y se discuten ampliamente y se aprueban en general y en particular por unanimidad. Leído el estatuto que entra en vigencia en el momento de ser aprobado se procede a la elección de las autoridades". ${ }^{\text {I9 }}$

Por último, en la reunión siguiente, del 2 de noviembre, se autoriza al grupo administrativo a imprimir carnets y estatutos. La indicación de la impresión permite pensar que este estatuto de 1938 - que constaba de I5 capítulos y 54 artículos - sí fue considerado definitivo por sus integrantes.

Hay algunos pocos rasgos del documento que se pueden advertir por la redacción de las actas. Uno es que no se le podía prohibir a un socio la entrada al local social a menos que se hubiera acordado su expulsión previamente.

18. Aníbal Lomba, Peña Pacha Camac 1932-1957. Una peña nacida en Boedo para toda la Ciudad, 30 .

19. Archivo Junta de Estudios Históricos del Barrio de Boedo, Actas, Peña Pacha Camac, is de octubre de 1938 . 
Otro es la estipulación de que, cada determinado tiempo, se tenía que realizar la convocatoria a la Asamblea General Ordinaria. También se fijaba que los socios, después de que pasara cierto periodo desde su afiliación, podían subir a la categoría "A".

\section{Los antecedentes del Teatro Popular José González Castillo}

En un principio, el teatro no figuraba entre las actividades de la Peña Pacha Camac. De hecho, el 28 de septiembre de 1932, se crearon subcomisiones de trabajo auxiliares a la Comisión Directiva y éstas eran: "de conferencias y de revista oral", "de exposiciones", y "de fiestas". No obstante, al poco tiempo, el 6 de octubre de 1932, se planteó hacer un festival artístico a beneficio de los artistas miembros de la institución que estaban viviendo una situación precaria. La propuesta se aprobó (con la salvedad de que el beneficiario fuese la Caja de Socorros de la propia institución). Existe la posibilidad de que en ese festival haya habido un primer intento de representación teatral, pero no lo sabemos a ciencia cierta.

Otro indicio de que la actividad teatral estaba rondando en las ideas de los integrantes de la peña, se puede ver en un aguafuerte que Roberto Arlt publicó el 22 de octubre del mismo año en el diario El Mundo. Allí, Arlt habla de la Peña de Artistas Pacha Camac y transcribe estas palabras de González Castillo: "Nosotros no sońábamos en tal éxito. [...] Ahora sí que podemos pensar en organizar un teatro aquí en esta terraza, que esté libre de las terribles exigencias de la taquilla”. ${ }^{20}$ Estas últimas palabras dan cuenta de la pretensión de González Castillo de llevar a cabo, en la peña, tareas más cercanas al incipiente teatro independiente, y distintas de las que realizaba con el teatro profesional. Como reflexiona Villa, la actividad de González Castillo al frente de la Peña Pacha Camac contrastó "con su producción teatral, que fue conformista, respondiendo a las demandas del teatro comercial popular". ${ }^{21}$

Al poco tiempo, el 5 de diciembre de 1932, quedó registrado en actas lo siguiente:

20. Citado en Roberto Arlt, "Peña de artistas en Boedo", El Mundo, secc. Aguafuertes porteñas, 22 de octubre, 1932, s.p.

2. Villa, José González Castillo. Militante de lo popular, 147. 
Cuadro Filodramático de la Peña Pacha Camac. El secretario, señor Lío, informa del ofrecimiento del presidente de la institución de un cuadro filodramático preparado por él para dar funciones a su oficio y nombre de la peña que llevará el nombre de ésta y cuya dirección nomina el señor Castillo, agregando el secretario señor Lío, que en el caso de aceptarse el ofrecimiento, el cuadro estaría en condiciones de dar su primer función en el teatro Boedo, el día i6 del corriente. Se aprueba por unanimidad el ofrecimiento. ${ }^{22}$

Como se verá, el nombre que se propone para el grupo es el mismo del de la peña. Pero Marial ${ }^{23}$ manifestó que, antes de tomar el nombre de la peña, el grupo filodramático se había llamado Las Cigarras. Lo mismo se informó en la revista Conducta, en noviembre de 1938, cuando se anunció la I $\mathrm{I}^{\mathrm{a}}$ Exposición de Teatros Independientes ${ }^{24}$ y se publicó una reseña de cada uno de los grupos participantes: "En 1932, José González Castillo, alma de Pacha-Camac, fundó el primer cuadro escénico, dándole el nombre de 'Las cigarras' para cambiarlo luego por el de Pacha-Camac, propio de la Agrupación". ${ }^{25}$ Si bien esa nota no tiene firma, por algunas otras de sus frases, se infiere que fue escrita por un integrante de la redacción de la revista más que por un miembro del teatro. Por lo tanto, surgen dudas sobre el momento en que el cuadro se llamó así. Es decir, en el acta dice que el cuadro filodramático realizará su primera función, por lo que sería llamativo que ya hubiese estado funcionando desde antes con otro nombre y que esto no hubiera quedado registrado en actas. Una posibilidad es que el nombre de Las Cigarras se haya utilizado después (en algún momento entre 1933 y 1937, del cual no tengo actas), aunque también sería extrańo que ambas publicaciones lo hubieran ubicado antes, ya que supongo que les debería haber sido más fácil rastrear los datos más novedosos que los más antiguos. Otra opción es que el cuadro filodramático nunca se haya llamado Las Cigarras, que el error original se haya dado en Conducta y que Marial lo haya retomado en su libro.

22. Archivo Junta de Estudios Históricos del Barrio de Boedo, Actas, Peña Pacha Camac, 5 de diciembre de 1932.

23. Marial, El teatro independiente.

24. Fue la primera actividad colectiva del movimiento de teatros independientes. Aparte del grupo de Boedo, participaron el Teatro del Pueblo, el Teatro Juan B. Justo y el Teatro Íntimo de la Peña.

25. Anónimo, " $\mathrm{I}^{\mathrm{a}}$ exposición de teatros independientes", Conducta. Al Servicio del Pueblo, núm. 4 (noviembre 1938): 4 . 
La función anunciada para el Teatro Boedo se llevó a cabo, aunque su desarrollo generó sospechas sobre González Castillo. Así, el 29 de diciembre, se registró en actas:

Enseguida usa de la palabra el señor Castillo para referirse extensiva y minuciosamente a los fines que ha perseguido al ofrecer a la institución el cuadro filodramático a que se refiere el acta anterior siendo el dar mayor brillo a la peña, como también el de colaborar en las funciones que puedan hacerse en beneficio de la misma agregando que para evitar suspicacias que fatalmente se despiertan va a demostrar ampliamente que él solamente ha sido perjudicado en sentido económico por la función dada en el teatro Boedo, no así desde otros puntos de vista, dejando en el seno de la comisión el convencimiento de que el seńor Castillo cubre con exceso las esperanzas cifradas en su nombre al ser elegido presidente de la institución. ${ }^{26}$

Gracias a esta problemática, González Castillo presentó su renuncia en enero de 1933, como ya se había enunciado, pero esta petición fue discutida y luego rechazada.

En la peña, las funciones del elenco teatral comenzaron en 1933. González Castillo era el director de los espectáculos y, entre otros, integraban el grupo Saulo Benavente (quien luego se convertiría en uno de los escenógrafos más destacados de la Argentina), Armando Clavier, Fernando Aló, José Vaccaro, Blanca Saavedra, y Alicia y Nelly Míguez Saavedra. En I934, el cuadro escénico contaba con 24 integrantes. Ya había representado Jazz, de Pagnol; El viaje sin regreso, de Sutton Vane; Carina, de Cromelink; La enemiga y Retazo, de Niccodemi; Canción de cuna, de María Lejarraga (primero, circuló bajo la autoría de Gregorio Martínez Sierra); El pato silvestre, de Ibsen; y otras pequeñas obras de Strinberg, O’Neill, Dantas, Álvarez Quintero, Cocteau y Pompel. Y según la nota "La Peña de Boedo honra al barrio y prestigia al país", ${ }^{27}$ el elenco se encontraba ensayando, en ese momento, Ten minutes alibi, pieza policial que se había estrenado hacía poco en el famoso Teatro Odeón. El texto también daba cuenta de los distintos lugares por los que había pasado el elenco: la

26. Archivo Junta de Estudios Históricos del Barrio de Boedo, Actas, Peña Pacha Camac, 29 de diciembre de 1932 .

27. Anónimo, "La Peña de Boedo honra al barrio y prestigia al país", Noticias del Sud, 6 de octubre, 1934, s.p. 
Biblioteca Ameghino de Luján, la Fraternidad Ferroviaria, y los clubes sociales José M. de Estrada y San José de Flores.

El vínculo entre la peña y el teatro profesional estuvo dado desde el comienzo, ya que González Castillo provenía de este ambiente. Sus sainetes habían sido estrenados en el Teatro Argentino (Entre bueyes no hay cornadas, Compañía Florencio Parravicini, 1908), el Teatro Moderno (Luigi, Compañía Enrique Muiño, 1909), el Teatro Buenos Aires (Los dientes del perro, Compañía Nacional Muińo-Alippi, 1918) y el Teatro Comedia (Chirimoya, 1930), entre otros. $\mathrm{El}$ artista formaba parte de ese mundo, siempre conectado con lo popular:

Natalín o la fonda del Pinchazo eran frecuentados por los puesteros del mercado vecino mezclados con escritores, periodistas, músicos populares, escultores, políticos y deportistas. Entre ellos, Florencio Sánchez, José Ingenieros, Agustín Riganelli, Alfredo L. Palacios, Antonio de Tomaso, José González Castillo, Florencio Parravicini, Jorge Newbery. ${ }^{28}$

De hecho, del ya mencionado Gran Festival Artístico que se hizo en el teatro Boedo, en diciembre de 1932, para celebrar el surgimiento de la peña, habían participado artistas profesionales, como María Padín, Enrique de Rosas, Matilde Rivera y Pedro Aleandro. Esa noche también actuó el primer actor cómico Pepe Arias. No obstante, en esa oportunidad, no habían cobrado por su trabajo, como solían hacer, sino que todo lo recaudado quedó a beneficio de la peña.

González Castillo continuó estrenando, en forma paralela a su labor en Boedo, sus obras en el teatro profesional. Incluso algunas fueron representadas en ambos circuitos. Beatriz Seibel ${ }^{29}$ sostiene que Pirincho el hijo del bosque fue llevada a escena, en 1934, por la compañía de Camila Quiroga en el Teatro Corrientes; que en 1936 se repuso en el Coliseo dirigida por González Castillo; y que, un año más tarde, se presentó en la peña - y en gira- con escenografía de Saulo Benavente y música de Cátulo Castillo (el hijo del fundador de la peña, quien fue un reconocido poeta y compositor de tango). Otra versión indica que el "Teatro ejemplar para el niño" — ciclo en el que estaba Pirincho el hijo del bosque — fue originado en la peña por los mismos actores que con-

28. Villa, José González Castillo. Militante de lo popular, I6I.

29. Beatriz Seibel, Historia del teatro argentino II 1930-1956: crisis y cambios (Buenos Aires: Corregidor, 20I0). 
formaban el conjunto teatral..$^{30}$ Este lazo, sin embargo, fue perdiéndose cuando el conjunto se transformó en un teatro independiente. ${ }^{3 \mathrm{I}}$

El cuadro filodramático de la peña no era un cuadro tradicional, que sólo replicara obras que se veían en el teatro profesional y que tuviera como primer objetivo entretener al público. Si bien eso sucedió con algunas obras - como por ejemplo La mala reputación, del propio González Castillo, que se hizo en el barrio de Boedo en I933, pero que ya se había estrenado en la escena profesional en 1920-, tuvo un repertorio variado, que incluyó a Eugene O'Neill, Henrik Ibsen, Maurice Maeterlink, Federico García Lorca, George Bernard Shaw, Florencio Sánchez, Armando Discépolo, Alejandro Berrutti, S. y J. Álvarez Quintero, Jacinto Benavente, Jean V. Pelerin, J. Camargo, Roberto Cayol y Carlos Mauricio Pacheco, entre otros. Empero, a diferencia de las decisiones que tomaba Leónidas Barletta en su Teatro del Pueblo, González Castillo adaptaba él mismo las obras "para que fueran más comprensibles ya que el público no estaba acostumbrado al drama de ideas" ${ }^{32}$ En la misma línea, el director y dramaturgo daba referencias previas a los espectadores sobre las obras que se iban a representar, "buscando de tal modo una comprensión cabal del texto por parte del auditorio". 33

El género que más transitó González Castillo como autor fue el sainete - en varios de sus estilos-, una de las variedades más de moda en el teatro profesional que tanto defenestraba Barletta. Villa advierte esta disidencia y sostiene que el pensamiento del fundador del Teatro del Pueblo "se oponía al de González Castillo, que en 1937 veía en el sainete no sólo 'un medio natural de expresión, en lo que el teatro tiene de comunicación colectiva, de relación simpática

30. Véase Lomba, Peña Pacha Camac 1932-1957.

31. Cuando el Teatro Popular José González Castillo fue invitado a formar parte de la I Exposición de Teatros Independientes se aclaró, en Conducta, que el grupo había comenzado a excluir "en lo posible la colaboración de profesionales"; Anónimo, " $\mathrm{I}$ a exposición de teatros independientes", Conducta. Al servicio del pueblo, núm. 4 (noviembre 1938): 4 . Es posible entender este hecho como una demostración de pertenencia al movimiento de teatros independientes. Empero, no se puede dejar de lado que algunos de sus miembros, como fue el caso de Saulo Benavente, ya estaban trabajando de forma paralela en el otro circuito. Por ejemplo, Benavente realizó la escenografía de la obra Mujeres, en 1939, presentada por la compañía de Mecha Ortiz, como da cuenta Cora Roca, Saulo Benavente. Escritos sobre escenografía (Buenos Aires: Instituto Nacional del Teatro, 20I3).

32. Villa, José González Castillo. Militante de lo popular, 165.

33. Lomba, Peña Pacha Camac I932-1957, 26. 
primigenia entre el creador y el espectador' sino 'nuestro auténtico medio de expresión teatral'”. ${ }^{34}$

A pesar de estas distancias, ambos teatristas tenían en común sus fuertes lazos con el barrio de Boedo y la cultura que se generó allí. Así, Leónidas Barletta fue invitado a dar una conferencia en la peña, que versó sobre la polémica literaria de Boedo y Florida. Otros invitados que acercó González Castillo a la peña fueron referentes del campo teatral profesional, como Vicente Martínez Cuitińo, Enrique García Velloso o Alberto Vacarezza —este último fue uno de los enemigos públicos de Barletta. Una foto expuesta en el libro Los productores. Historias de empresarios teatrales argentinos de todos los tiempos dio cuenta del hecho. Allí se ve a un nińo leyendo una pizarra colocada en la vereda del teatro con el siguiente texto: "Pacha Camac. Sábado I6 a las 22 hs. hablará el conocido autor Alberto Vacarezza. Consejos que se le olvidaron al Viejo Vizcacha. A continuación: Teatro. Música. Canto. Entrada libre". ${ }^{35}$ Los consejos que se le olvidaron al Viejo Vizcacha hacían referencia a los que daba el personaje del Viejo Irala en La biblia gaucha, publicada en 1936. Vacarezza y el director de la Pacha Camac no sólo fueron compañeros de trabajo - escribieron en colaboración (junto a Alejandro Berruti) Mañana será otro día, obra que se estrenó en 1930-, sino que también fueron muy amigos. De hecho, Vacarezza fue uno de los oradores que - con Rodríguez Acasuso, Martínez Cuitińo, Quiroga, Zucchi y Canaro- despidió los restos de González Castillo en el cementerio.

\section{El surgimiento del Teatro Popular José González Castillo}

La primera acta de 1937 que tengo tiene fecha del 30 de octubre. En ella, los socios propusieron, en Asamblea Extraordinaria, reorganizar la institución debido a la muerte de González Castillo, sucedida hacía apenas ocho días. Benavente, quien colaboraba con la escenografía de la peña desde sus tempranos i6 años, tenía entonces 2I y fue quien tomó la palabra. Dijo que, más que nunca, se necesitaba de la colaboración de todos los socios para sostener la peña y

34. Villa, José González Castillo. Militante de lo popular, I49.

35. Gabriela Kogan, Marcela López, Carlos Ulanovsky y Susana Pelayes, Los productores: historias de empresarios teatrales argentinos de todos los tiempos (Buenos Aires: AADET, Asociación Argentina de Empresarios Teatrales, 20I2), 78-79. 
continuar la tarea iniciada por su fundador. La administración que él propuso para su reorganización era "una forma comunitaria de gobierno" . ${ }^{66}$ Así, la nueva Junta Ejecutiva estaría compuesta por dos consejeros, un secretario, un prosecretario, un tesorero, un protesorero y cuatro grupos de artistas especializados en artes plásticas, artes escénicas, música y literatura. Esta disposición se aprobó. Benavente fue elegido secretario general y también integró la comisión de artes escénicas, junto a Arturo Mario (director), Mario Cozzani, Cuca Guivás, Blanca Saavedra, José M. Saftich y José J. Vaccaro.

La primera acción de las nuevas autoridades, con relación al teatro, fue autorizar al grupo escénico para que actuara en un festival organizado por la Universidad Popular de Boedo, en los salones de la Sociedad Balear.

En la siguiente reunión, ya a cargo de la nueva comisión, realizada el 8 de noviembre de 1937, Benavente "hace moción para que el grupo de arte escénico se constituya en entidad autónoma bajo la denominación de Teatro Popular José González Castillo". ${ }^{37}$ Esta iniciativa fue aprobada con dos condiciones: que todos los actos del Teatro Popular José González Castillo fueran autorizados y fiscalizados por la Comisión Directiva de la Peńa Pacha Camac; y que los posibles réditos económicos que se obtuvieran con sus funciones fueran a beneficio de la peña. Seibel ${ }^{38}$ señala que quienes quedaron a cargo de la dirección artística del grupo fueron Arturo Mario y Atilio Supparo.

Como he anticipado, José Marial había afirmado que la autonomía del grupo de teatro dentro de la peńa se dio en 1934. Si bien no cuento con las actas de dicho año, el párrafo antes mencionado corrobora que la constitución en entidad autónoma se dio en 1937, al mismo tiempo que el grupo modificó su nombre.

En línea con esta independencia otorgada al grupo de teatro, el is de agosto de I938, se resolvió que el Teatro Popular José González Castillo llevara un libro de actas con sus sesiones.

Asimismo, dos meses después, en la Asamblea Extraordinaria, donde la Junta Ejecutiva Reorganizadora expuso el balance anual, se afirmó:

36. Lomba, Peña Pacha Camac 1932-1957. Una peña nacida en Boedo para toda la Ciudad, 32.

37. Archivo Junta de Estudios Históricos del Barrio de Boedo, Actas, Peña Pacha Camac, 8 de noviembre de 1937 .

38. Beatriz Seibel, Historia del teatro argentino II 1930-1956: Crisis y cambios (Buenos Aires: Corregidor, 20IO). 
Teatro Popular: En noviembre de 1937, instituyóse en Teatro Popular Independiente el cuadro escénico que hasta entonces y durante 5 años consecutivos había actuado en el seno de Pacha Camac. Diósele el nombre de "José González Castillo" en homenaje del que fuera su fundador, director y maestro. Efectuáronse representaciones a precios populares y la sola afluencia de público a sus espectáculos vino a certificar que su existencia llenaba una verdadera necesidad en el barrio. ${ }^{39}$

Estas palabras terminan de confirmar que la autonomía del grupo dentro de la peña y su constitución como grupo de teatro independiente datan, en efecto, del 8 de noviembre de 1937.

En las asambleas de la peña, el grupo de arte escénico estaba representado por una comisión de tres miembros que se renovaba cada año. ${ }^{40} \mathrm{~A}$ su vez, tenía sus propias autoridades: un director, dos asesores, una comisión de lectura y los responsables del curso de arte escénico y declamación. ${ }^{4 \mathrm{I}}$

En noviembre de 1939, el conjunto de arte escénico realizó la experiencia de convocar a aficionados al teatro a inscribirse en el elenco estable. No obstante, esta medida no fue satisfactoria, y el cuadro continuó constituido por los antiguos actores y por aquellos que habían sido tomados del curso de arte escénico. Al respecto, debo señalar que la Peña Pacha Camac fue pionera en lo referente a la formación de los actores. Si bien fue recién en el acta del Io de junio de 1938 cuando se resolvió crear un curso de ensayo de arte escénico, desde los inicios de la peña había estado presente su "espíritu docente". En este sentido,

39. Archivo Junta de Estudios Históricos del Barrio de Boedo, Actas, Peńa Pacha Camac, Is de octubre de 1938 .

40. Comisión de arte escénico elegida el is de octubre de I938: Juan Campos, Blanca Saavedra y Carmen Méndez; Comisión de A. E. elegida el 25 de noviembre de 1939: Blanca Saavedra, Cuca Quirós y Mario del Carpio. A los pocos días, el 29 de noviembre, se aclara que Alicia Míguez Saavedra queda como secretaria especial del área de arte escénico y Carlos Trotta, como encargado del curso de arte escénico. Antes de cumplir su mandato (3/8/40), Mario del Carpio presenta su renuncia al cargo de miembro de la comisión de arte escénico, y es reemplazado por Nino Graciani; Comisión de A. E. elegida el I6 de noviembre de 1940: Mario Bellomo, Mario del Carpio e Ismael Esteban; Comisión de A. E. elegida el I5 de noviembre de 194I: Nino Graciani, Tito de George y Mario Bellomo; Comisión de A. E. elegida el 28 de noviembre de 1942: Luisa Manghi, Mario Bellomo y Nino Graciani.

4I. El I5 de agosto de 1938, se informan las autoridades nombradas por el grupo de arte escénico: director, señor Lodolini; asesores, C. Quirós y N. Graciani; comisión de lectura: Luisa Manghi, José Saftid y A. Gacbani; curso de arte escénico y declamación; director: Juan Campos; asesor: E. Burruni; reemplazante: O. Trotta. 
Lomba manifiesta que su claro propósito era "abrir las puertas de la cultura e instrucción a una amplia franja de la población que carecía de guías adecuados para introducirse en ella". ${ }^{42} \mathrm{~A}$ finales de ese año, en el tradicional balance que hacía la institución cada ańo, se mencionó este acontecimiento fundamental para la organización teatral del grupo de la peña: "se creó un curso de aplicaciones de arte escénico y declamación con miras de convertirlo en un futuro próximo en una verdadera escuela dependiente del Teatro Popular". ${ }^{43}$ En este sentido, Marial sostiene que el Teatro Popular José González Castillo tuvo "el incuestionable mérito de haber sido uno de los primeros en implantar los cursos de capacitación de actores, estructurándose de esta manera una incipiente escuela de arte escénico". ${ }^{44} \mathrm{El}$ grupo precursor tenía planes ambiciosos respecto al tema: en 1942 los profesores del curso de arte escénico (Mario del Carpio y Luisa Manghi) organizaron un nuevo plan de estudios que requería cinco horas semanales para los alumnos. No obstante, estas ideas fueron difíciles de cumplir debido, principalmente, a las obligadas mudanzas que tuvieron que transitar.

$\mathrm{Al}$ retomar el desarrollo del conjunto de arte escénico, se observa que, en diciembre de 1939, se propuso buscar un director artístico que orientara ${ }^{45}$ al cuadro escénico de la peña. Si bien, en una primera instancia, se había resuelto convocar a Miguel Mileo para hacerse cargo de la dirección, en abril se informó que las gestiones realizadas no habían arrojado resultados. Esto llevó a atrasar el inicio de la temporada — planeada para marzo de 1940- hasta la segunda quincena de mayo.

En febrero de 1940, la comisión de arte escénico solicitó que se nombrara una subcomisión, integrada por socios de la agrupación, para que secundara sus funciones. Tal comisión colaboradora quedó integrada por Saftich, Benavente, Luisa Menghi, Angélica Sacobe y Carmen Méndez. En noviembre del mismo año, se creó, por iniciativa de Mario del Carpio, otra comisión, esta vez de lectura, formada por cuatro miembros (uno del grupo administrativo, uno del grupo de literatura, uno del grupo de arte escénico y un representante de los actores), para que se encargara de la lectura y la aprobación de las obras a

42. Lomba, Peña Pacha Camac 1932-1957. Una peña nacida en Boedo para toda la Ciudad, II.

43. Peña Pacha Camac; Actas; is de octubre de 1938; Archivo Junta de Estudios Históricos del Barrio de Boedo.

44. José Marial, El teatro independiente, II9.

45. En el encuentro posterior, cuando se corrige esta acta, se modifica la palabra "oriente" por "dirija" (Archivo Junta de Estudios Históricos del Barrio de Boedo). 
representar. Esto generó discusiones: "Benavente pide se aclare la situación del Teatro Independiente, que él considera separado del grupo A. E. [de arte escénico] y presenta un proyecto: que se nombre otra comisión de cuatro miembros sin especificación de los cargos que desempeñen, para que con el grupo A. E. tengan a su cargo las representaciones del Teatro Popular José González Castillo, pero no de las que él considera de la peña en sî". ${ }^{46}$ A continuación, Del Carpio amplió su propuesta uniendo ambas actividades — "el teatro de la peña y el Teatro Popular"47 — en la comisión que recién se había nombrado y en el grupo de arte escénico. Las condiciones serían las ya mencionadas. Después de un prolongado debate, se votó y aprobó el proyecto de Del Carpio. La nueva comisión asesora quedó integrada por $\mathrm{S}$. Benavente (grupo administrativo); A. Sarole (grupo de literatura), Del Carpio (grupo de arte escénico), y un representante de los actores que sería elegido posteriormente..$^{8}$

Si bien no terminan de quedar claras cuáles eran las diferencias entre el grupo de arte escénico y el Teatro Popular José González Castillo que marcó Benavente, se infiere, por el análisis de las actas, que ambos conjuntos estaban compuestos por las mismas personas y que, en tal caso, lo que variaba era el lugar que ocupaba la peña. Es decir, deduzco que había representaciones que eran "de la peña en sî", como dijo Benavente, y otras que contaban con una mayor independencia. En este sentido, se entiende que las funciones —que se realizaban dos veces por semana con gran respuesta del público—ya no se constituían como un complemento de las actividades de la peña, sino que, como se manifiesta en la revista Conducta, "por sí solas alcanzaban un fin determinado". ${ }^{49}$ No obstante, creo que el Teatro Popular José González Cas-

46. Archivo Junta de Estudios Históricos del Barrio de Boedo, Actas, Peña Pacha Camac, 21 de noviembre de I940.

47. Archivo Junta de Estudios Históricos del Barrio de Boedo, Actas, Peña Pacha Camac, 2 I de noviembre de 1940 .

48. En la sesión del I8 de enero de 194I se comunicó que Luisa Manghi había resultado electa por los actores y que Burruni reemplazaría a Benavente debido a su renuncia. El 3 de diciembre de $194 \mathrm{I}$ la comisión de lectura de teatro quedó formada por Tito de George (grupo de arte escénico), Eduardo Burruni (administración) y Juan C. Buononi (grupo de literatura). La comisión se completó al mes siguiente con Sergio, delegado de los actores. El 5 de diciembre de 1942 la subcomisión de lectura (así expresada) quedó conformada por Eduardo Burruni (grupo administrativo), Luisa Manghi (grupo de arte escénico), Juan Carlos Burruni (grupo de literatura) y Fernando Aló (actores). Este último renunció en enero de 1943 y fue reemplazado por Horacio Delfino.

49. Anónimo, " $\mathrm{I}^{\mathrm{a}}$ exposición de teatros independientes", Conducta. Al servicio del pueblo, núm. 4 (noviembre i938): 4 . 
tillo era el grupo de arte escénico de la Peña Pacha Camac y que sus términos se utilizaban indistintamente en la escritura de las actas.

Desconozco si la resolución del debate no contentó a Benavente, pero lo cierto fue que faltó a la reunión consecutiva y, en la siguiente, dimitió. Así, el II de enero de 194I, el hasta entonces secretario general presentó su renuncia a todos los cargos que desempeñaba en la institución y, acto seguido, se retiró. Este hecho repentino generó que se pactara una sesión especial para tratar el tema y una comisión para que intentara convencerlo de retractarse. Esto no fue posible y, el i8 de enero, se aceptó la renuncia de Benavente, quien ya había retirado de la peña algunos trajes y armaduras de su propiedad. La secretaría general fue asumida por el prosecretario Burruni.

\section{Palabras finales}

A lo largo de este trabajo, he dado cuenta del surgimiento del Teatro Popular José González Castillo — un destacado grupo de teatro independiente, sito en el barrio de Boedo, lejos del tradicional centro de Buenos Aires - a partir del análisis de su documentación original. Considero que volver a las fuentes es de vital importancia para los estudios históricos del teatro independiente, práctica teatral que, aún en la actualidad, se desarrolla con vital importancia en la capital de la Argentina.

Además, se advierte que conocer de primera mano los documentos permite derribar mitos o confusiones que se hayan arrastrado a lo largo de los ańos, como también dar cuenta de las particularidades de cada teatro independiente. En este sentido, se entiende que el contexto de creación de cada uno puede ser determinante para su desarrollo.

La Peña Pacha Camac, cuna del Teatro Popular José González Castillo, dejó de funcionar en la década de los años cincuenta, luego de sufrir varias mudanzas y serias dificultades — a lo largo de los años - para instalarse en un lugar, después de que la expulsaran de su sitio original, en 1938, a raíz de la venta del Café Biarritz a la municipalidad (que quería ampliar el Banco Municipal de Préstamos, actual Banco Ciudad). ${ }^{50}$ Sin embargo, vale la

50. Además, la Peña Pacha Camac nunca logró que la municipalidad le cediera un terreno. Otros grupos de teatro independiente, como el Teatro del Pueblo, tuvieron más suerte. El periodista Edmundo Guibourg, desde el diario Crítica, el 31 de mayo de 1938, dio cuenta de esta 
pena recuperar su valiosa historia, tanto para la comunidad de Boedo — que la mantiene presente, sobre todo, gracias al trabajo de la Junta de Estudios Históricos del Barrio de Boedo-, como para los estudios del teatro independiente. 's

desigualdad: "Un mal día reciente llegó allí la piqueta municipal, a demoler como consecuencia de la adquisición de un viejo edificio, y no se tuvo en cuenta para nada, en absoluto, que se dejaba sin sede a una institución popular, por muchos conceptos meritoria. [...] No es extraño, entonces, que obligue a la suspicacia el considerar con qué asombrosa facilidad el llamado Teatro del Pueblo obtuvo de la pasada administración municipal tan desproporcionados favores. [...] Mientras que por un lado se despilfarran centenares de miles de pesos, lo bastante para subvencionar a todas las compañías teatrales del país, y ello al solo objeto de asegurar el desenvolvimiento de un núcleo de aficionados, por el otro se conspira contra la existencia de organizaciones de propósitos artísticos que son más especial y más genuinamente populares"; citado en Mónica Villa, José González Castillo. Militante de lo popular, I66. 